\title{
A randomised double blind controlled crossover trial of taurine supplementation in home parenteral nutrition
}

\author{
A. Culkin ${ }^{1}$, A. M. Madden ${ }^{2}$, A. Forbes ${ }^{3}$ and S. M. Gabe ${ }^{1}$ \\ ${ }^{1}$ Lennard-Jones Intestinal Failure Unit, St Mark's Hospital, Harrow HA1 3UJ, UK, ${ }^{2}$ Dietetics, University of Hertfordshire, \\ Hatfield, AL10 9AB, UK and ${ }^{3}$ Clinical Nutrition and Gastroenterology, University College Hospital, London NWI 2BU, UK
}

Cholestatic liver disease is one of the main causes of death during home parenteral nutrition (HPN). Cavicchi reported that $65 \%$ of patients on HPN developed chronic cholestasis after a median of 6 months ${ }^{(1)}$. Taurine deficiency has been implicated in the development of parenteral nutrition associated cholestasis (PNAC) and studies in human subjects receiving HPN have shown low plasma levels which were restored using intravenous taurine supplementation ${ }^{(2)}$.

The aim of the present study was to determine if the inclusion of $1 \mathrm{~g}$ of parenteral taurine as part of the nitrogen source of HPN improves liver function. Patients on HPN identified as experiencing PNAC were invited to participate in the study. Chronic cholestasis has been defined as occurring when two of the three liver function tests [alkaline phosphatase, bilirubin and $\gamma$-glutamyl-transferase (GGT)] are persistently elevated for over 6 months to a value of 1.5 -fold the upper limit of normal ${ }^{(3)}$. Patients were randomised to receive taurine supplemented HPN or taurine free HPN. Patients were reassessed after 3 months and then entered a 1 month washout period where they received taurine free HPN. After this they were crossed over onto the alternate amino acid solution for a further 3 months and reassessed. Statistical analysis was performed by calculating the difference in results between the two periods for each subject and comparing between treatment orders using the unpaired $t$-test. The study was approved by the local ethics committee and all patients gave informed consent.

Eleven patients completed both arms of the study. No difference was seen between the groups at randomisation. The study showed that taurine supplemented HPN when compared to taurine-free HPN did not significantly improve liver function (see table). Nutritional status remained constant and no difference in complication rates was observed during the study.

Table 1 Treatment effect (Taurine - no taurine, $95 \% \mathrm{CI}$ )

\begin{tabular}{lccc}
\hline & Alkaline phosphatase $(\mathrm{IU} / \mathrm{l})$ & Bilirubin $(\mu \mathrm{mol} / \mathrm{l})$ & $\mathrm{GGT}(\mathrm{IU} / \mathrm{l})$ \\
\hline Baseline & 234 & 18.4 & 252 \\
Treatment effect Taurine - no taurine & $-8(-55,39)$ & $5.7(-9,20.5)$ & $14(-31,59)$ \\
$P$ value & 0.69 & 0.38 & 0.48 \\
\hline
\end{tabular}

The study has shown that intravenous taurine does not appear to improve liver function in patients with PNAC over this time frame. Intravenous taurine can be given for 3 months without any adverse effects.

1. Cavicchi M, Beau P, Crenn P, et al. (2000) Ann Intern Med 132, 525-532.

2. Kopple JD, Vinton NE, Laidlaw SA et al. (1990) Am J Clin Nutr. 52, 846-853.

3. Nanji AA \& Anderson FH (1985) J Parenteral Enteral Nutr 9, 307. 